\title{
Geiger mode APD's for the underground cosmic ray experiment EMMA
}

\author{
L. Bezrukov ${ }^{a}$, K. Butin ${ }^{a}$, I. Davitashvili ${ }^{a}$, I. Dzaparova ${ }^{a}$, T. Enqvist ${ }^{b}$, H. Fynbo ${ }^{c}$, \\ L. Golyshkin ${ }^{a}$, Zh. Guliev ${ }^{a}$, L. Inzhechik ${ }^{a}$, A. Izmaylov ${ }^{a}$, J. Joutsenvaara ${ }^{b}$, \\ M. Khabibullin ${ }^{a}$, A. Khotjantsev ${ }^{a}$, Yu. Kudenko*a, P. Kuusiniemi ${ }^{b}$, \\ B. Lubsandorzhiev ${ }^{a}$, O. Mineev $^{a}$, V. Petkov ${ }^{a}$, R. Poleshuk ${ }^{a}$, T. Räihä ${ }^{b}$, J. Sarkamo ${ }^{b}$, \\ B.A.M. Shaibonov ${ }^{a}$, A. Shaykhiev ${ }^{a}$, W. Trzaska ${ }^{d}$, G. Volchenko ${ }^{a}$, V. Volchenko ${ }^{a}$, \\ A. Yanin ${ }^{a}$, N. Yershov ${ }^{a}$, D. Zykov ${ }^{a}$ \\ ${ }^{a}$ Institute for Nuclear Research of the Russian Academy of Sciences, 117312 Moscow, Russia \\ ${ }^{b}$ CUPP/Pyhäsalmi, University of Oulu, FIN-90014 Oulu, Finland \\ ${ }^{c}$ Department of Physics and Astronomy, University of Aarhus, DK-8000 Århus, Denmark \\ ${ }^{d}$ Department of Physics, University of Jyväskylä, FIN-40351 Jyväskylä, Finland \\ E-mail: bezrukov@inr.ac.ru, butin@inr.ru, dzaparova@yandex.ru, \\ timo.enqvist@oulu.fi, izmayloveinr.ru, inzhechikestream.ru, \\ pasi.kuusiniemi@oulu.fi, marateinr.ru, alex@inr.ru, kudenko@inr.ru, \\ oleg@inr.ru, vpetkov@yandex.ru, lubsand@pcbai10.inr.ruhep.ru, \\ juho.sarkamo@oulu.fi, shaykhiev@inr.ru, trzaska@phys.jyu.fi, \\ vvolchenko@yandex.ru, yanin@yandex.ru, yershov@inr.ru, zykov@inr.ru
}

Multi-pixel photodiodes operating in a limited Geiger mode will be used for photoreadout of scintillator counters in underground cosmic ray experiment EMMA. Main parameters of photodiodes and the performance of EMMA scintillator counters are presented.

International Workshop on New Photon Detectors PD09

June 24-26 2009

Shinshu University, Matsumoto, Japan

\footnotetext{
* Speaker.
} 


\section{Introduction}

The goal of the EMMA experiment is to study the chemical composition of the primary cosmic rays around the "knee" region at the energy of about $3 \times 10^{15} \mathrm{eV}$ by measuring the multiplicity, lateral distribution and arrival direction of the underground cosmic ray muons. The EMMA detector will be constructed in the Pyhäsalmi mine, Finland [1]. It consists of drift chambers and plastic scintillator detectors and has the total area of $\sim 135 \mathrm{~m}^{2}$ at the depth of $85 \mathrm{~m}$ (about 240 meters of water equivalent).

In total, about 1600 scintillator counters, $122 \times 122 \mathrm{~mm}^{2}$ and $3 \mathrm{~cm}$ thick, are arranged in $4 \times 4$ arrays, each of 16 counters, which form individual detectors of $50 \mathrm{~cm} \times 50 \mathrm{~cm}$ in cross section. Scintillator counters use wavelength shifting (WLS) fiber readout. Photosensors, Geiger-mode multi-pixel avalanche photodiodes, are mounted inside the counters. The set-up employing such photosensors is a unique device designed for the cosmic-ray induced underground muon detection.

\section{Parameters of MRS APD's}

Detailed description of multi-pixel avalanche photodiodes with a metal-resistor-semiconductor layer structure operating in a limited Geiger mode (hereafter referred to as MRS APD's or MRS photodiodes) can be found in Refs. [2,3]. Such a photosensor consists of many independent sensitive pixels produced on a common $\mathrm{p}$-type silicon substrate. A simplified topology of an MRS photodiode, invented and designed by the Center of Perspective Technologies and Apparatus (CPTA), Moscow, is shown in Fig. 1(a). Each pixel operates as an independent Geiger micro-counter with a

(a)

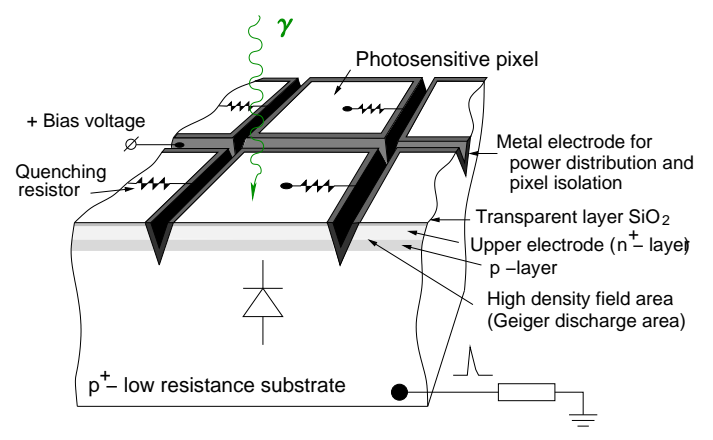

(b)

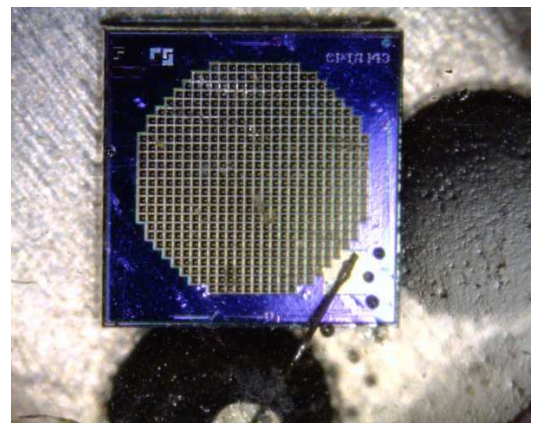

Figure 1: MRS APD: (a) schematic view of the structure; (b) photo of the sensitive area.

gain of the same order as a vacuum photomultiplier. Pixels are separated by grooves filled with an optically non-transparent material to suppress an optical cross-talk. The MRS photodiodes with 556 pixels of $35 \times 35 \mu \mathrm{m}^{2}$ each and total sensitive area of $1.1 \mathrm{~mm}^{2}$ (see Fig. 1(a)) were manufactured by CPTA for the EMMA experiment. Main parameters of these MRS APD's are reviewed below.

Bias voltage. The bias voltage, $V_{\text {bias }}$, applied on a photodiode creates a very high electric field in a very thin layer ( $\sim 1 \mu \mathrm{m}$ p-n junction). The bias voltage is set to be above the breakdown level, $V_{b d}$, so that the electric field can sustain the carrier's avalanche; however there is no current 
through the depletion layer until the first carrier is generated. Value of overvoltage $\Delta V=V_{\text {bias }}-V_{b d}$ determines the gain, photon detection efficiency (PDE), intrinsic noise and cross-talk. Typical bias voltage of MRS APD's for EMMA detectors is around 30-32 V. We apply the overvoltage of $4 \mathrm{~V}$ in operating mode for tests. EMMA electronics will automatically tune the bias voltages to have the background signals in the detector roughly at the same rate for all counters.

Photon detection efficiency. The PDE was measured at room temperature with a spectrophotometer and a calibrated PMT as described in $[4,5]$. Our result for PDE is about $25 \%$ for green light region (WLS fiber emission spectrum) at the operating bias voltage. The PDE values of MRS APD's in green light region is close to that of Hamamatsu MPPC's manufactured for the T2K experiment $[6,7]$. We have come to this conclusion after comparative measurements between MRS APD's and MPPC's with a reference light source.

Temperature dependence. Since the breakdown voltage depends on temperature the main parameters are sensitive to ambient temperature. Parameters of an MRS photodiode (PDE and gain) were measured inside a thermostatic box in a wide temperature interval from 10 to $38{ }^{\circ} \mathrm{C}$. A WLS Y11 fiber excited by a blue LED was used as a light source. The stability of LED light was measured to be better than $0.1 \% /{ }^{\circ} \mathrm{C}$. The results of the measurements are shown in Fig. 2. The MRS APD gain decreases with temperature as $-0.4 \% /{ }^{\circ} \mathrm{C}$, while the PDE varies with temperature as

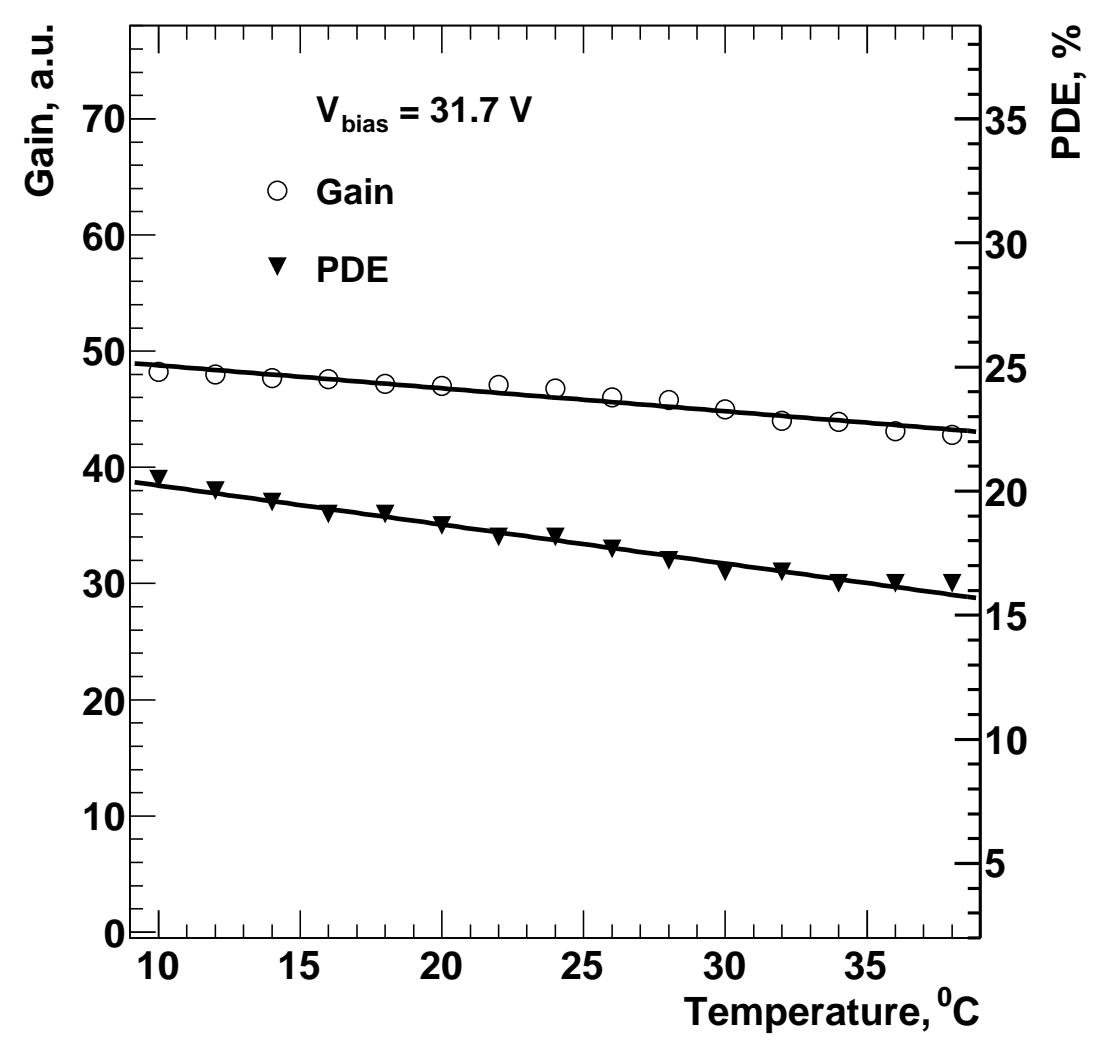

Figure 2: The photon detection efficiency for green light $(\lambda \sim 515 \mathrm{~nm})$ and the gain of an MRS APD as a function of the temperature. 
$-0.9 \% /{ }^{\circ} \mathrm{C}$. The weak sensitivity of MRS APD parameters to temperature can be explained by two factors: a weak temperature dependence of the breakdown voltage and a relatively high $\Delta V . V_{b d}$ was measured in the range from 10 to $35^{\circ} \mathrm{C}$. As seen from Fig. 3, breakdown voltage shows a weak

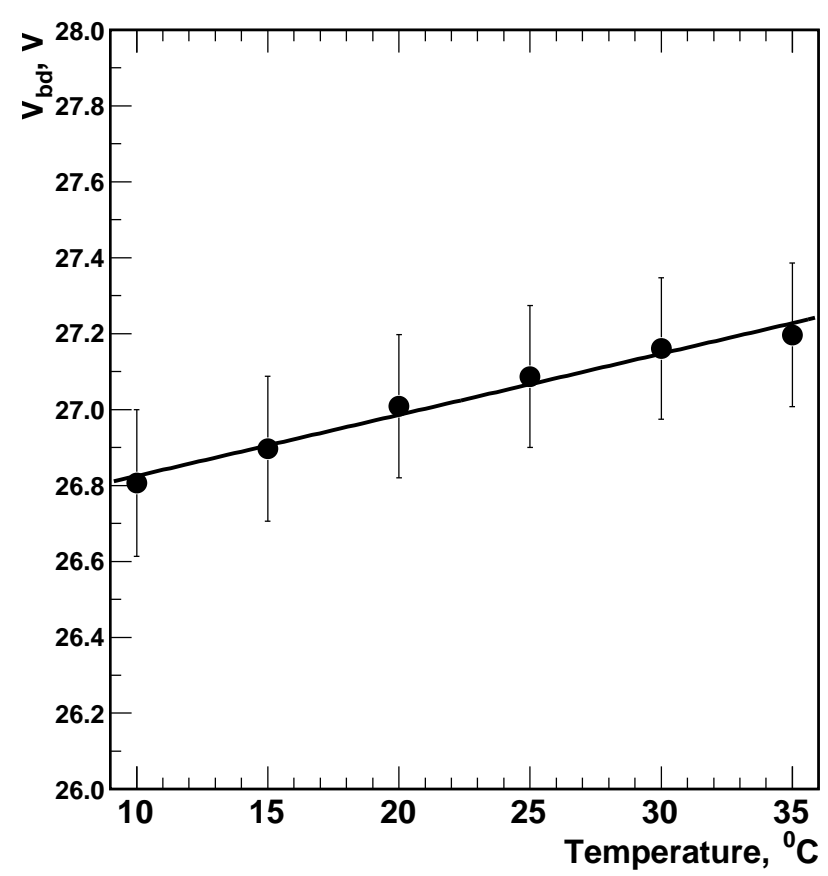

Figure 3: Temperature dependence of the breakdown voltage. The solid line shows the best fit with a gradient of $16 \mathrm{mV} /{ }^{\circ} \mathrm{C}$.

temperature dependence with a gradient of about $16 \mathrm{mV} /{ }^{\circ} \mathrm{C}$.

Pulse shape. The pulse shape of this type of photosensor is determined by a quenching resistor which is about $20 \mathrm{M} \Omega$. This value, which affects the pulse shape, was not well controlled during the production of wafers. The pulse shape of some MRS APD's has a long tail up to a few $\mu \mathrm{s}$, as discussed in Ref. [8]. To overcome such a problem, all MRS APD's were separated into 4 groups according to the ratio of the fast and slow (tail) components. In addition, a large quenching resistor causes the long recovery time of about $1 \mu \mathrm{s}$. Since the expected counting rate in the mine, including background from the surrounding rock, will be $\leq 100 \mathrm{~Hz}$ per a single counter [9], the recovery time is not a problem for the EMMA experiment. On the other hand, the long recovery time allows us to avoid after-pulses in contrast to MPPC's which were designed to have a fast recovery time [10].

In total, about 2200 MRS photodiodes fabricated from a few different wafers were tested. About $10 \%$ of delivered devices were rejected as defective (no signal) during preliminary tests and returned back to the manufacturer. No more dead MRS APD's were found during tests after acceptance. In order to meet the requirements of the experiment, the operating bias voltages were set so that the dark rate of each device was close to a reference value of about $1 \mathrm{MHz}$ at $22{ }^{\circ} \mathrm{C}$ at a discriminator threshold of 0.5 p.e. The breakdown voltages for these MRS APD's were found to be in a wide range $25-31 \mathrm{~V}$ that determines the range of $29-35 \mathrm{~V}$ for the applied bias voltages to provide the PDE value of $\geq 20 \%$ for green light. 
(a)

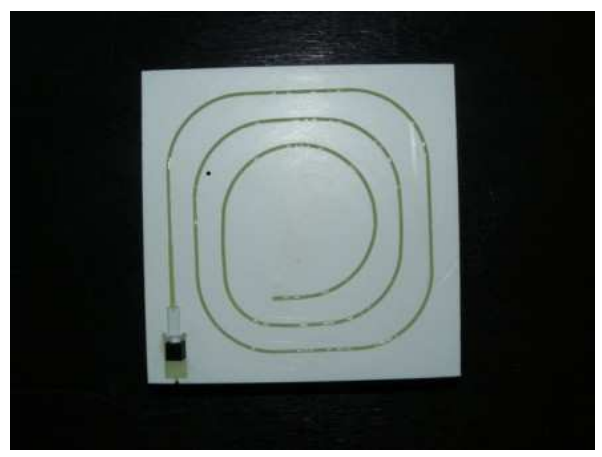

(b)

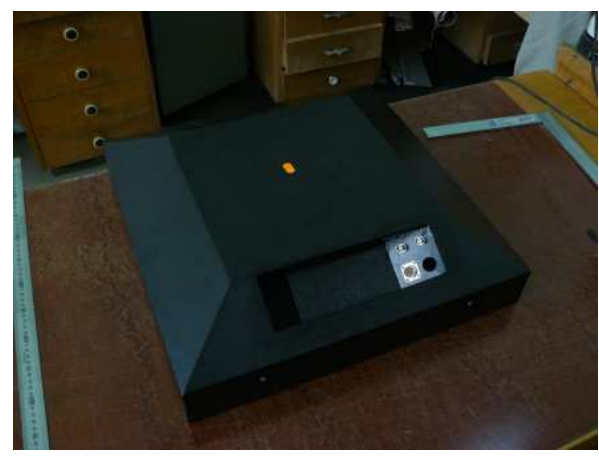

Figure 4: Photographs: (a) an individual scintillator counter with glued Y11 fiber and embedded optical connector; (b) an EMMA module comprised of 16 counters in a $4 \times 4$ assembly.

\section{EMMA counters}

An EMMA scintillator counter is shown in Fig. 4(a). Polystyrene scintillator tiles $(122 \times$ $122 \times 30 \mathrm{~mm}^{3}$ ) have been manufactured at IHEP, Protvino, Russia. Scintillating light is collected by a single Y11 (Kuraray) WLS fiber of $1 \mathrm{~mm}$ diameter glued into a spiral groove of $3.2 \mathrm{~mm}$ depth with the BC600 Bicron glue. The groove was carved by a Woodpecker engraving-milling machine of which speed and cutting tools were optimized to make a clean groove to provide the good transmission of the scintillating light through the cut surfaces. This technique was developed for scintillator counters for muon detection [11] in the near neutrino detector of the long baseline neutrino experiment T2K [12]. The fiber is viewed from one end by an MRS photodiode, the other end is covered by an aluminized mylar reflector. The MRS APD is placed inside an optical connector mounted in a small cut $\left(8 \times 8 \times 17 \mathrm{~mm}^{3}\right)$ made in the scintillator, as shown in Fig. 4(a). The stability of the optical contact between the fiber and MRS photodiode is provided by a foam spring. This readout configuration allows us to collect scintillation light uniformly over the entire scintillator. Outer surfaces of scintillators were etched by a chemical agent that resulted in the formation of a micropore deposit over the plastic surface. The thickness of the deposit $(30-100$ $\mu \mathrm{m}$ ), which works as a diffuse reflector, depends on the etching time. Details can be found in Ref. [13]. Scintillators with glued WLS fibers were wrapped into an additional reflector layer of $0.1 \mathrm{~mm}$ thick Tyvek paper that increases a light yield by about $15 \%$. One module is composed of 16 scintillator counters $\left(4 \times 4\right.$ assembly) which are packed into a steel box of $0.5 \times 0.5 \mathrm{~m}^{2}$, as shown in Fig. 4(b).

The performance of EMMA counters was tested with cosmic ray muons using a small $2 \times 2$ $\mathrm{cm}^{2}$ trigger counter placed at the center of a tested counter. A typical muon spectrum is shown in Fig. 5. The light yield of about 150 photoelectrons was obtained for a $6 \mathrm{MeV}$ energy deposited by cosmic muons in the $3 \mathrm{~cm}$ thick counter. The obtained time resolution $(\sigma)$ is about $0.9 \mathrm{~ns}$ (see Fig. 5) and mainly determined by the slow decay time (9-12 ns) of the Y11 fiber.

EMMA counters must provide a high light yield to separate cosmic muon signals from photon 

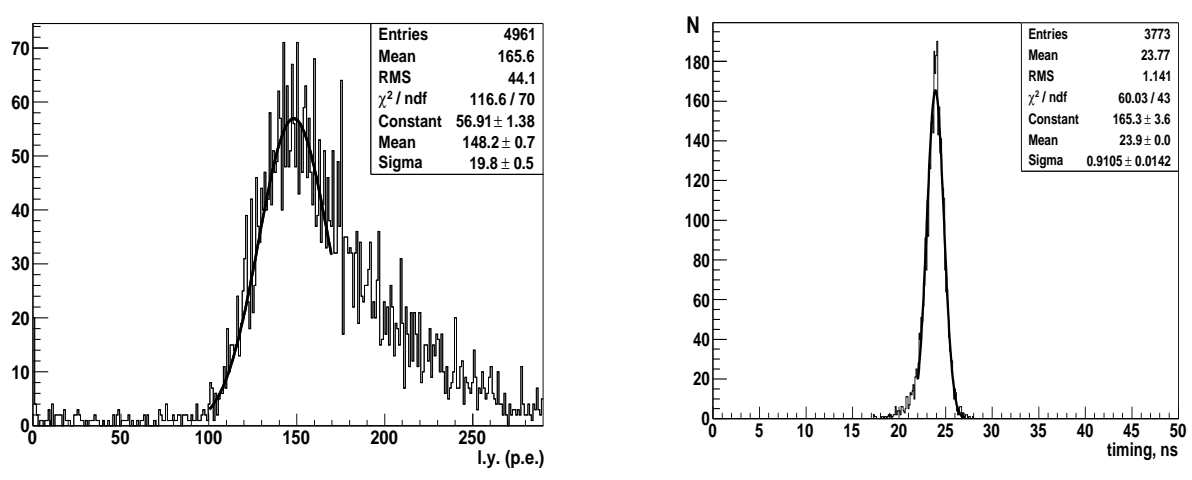

Figure 5: Left: the cosmic muon spectra measured by a typical counter. The light yield of 150 p.e. corresponds to the deposited energy of about $6 \mathrm{MeV}$. Right: time resolution for cosmic muons.

background of the rock walls $\left(E_{\gamma}=2.6 \mathrm{MeV}\right)$. The light yield of more than 100 p.e. per cosmic muon allows us to suppress the photon background and keep a high efficiency $(>95 \%)$ for cosmic muons. The light yield distribution for about 800 counters is shown in Fig. 6. Almost all man-

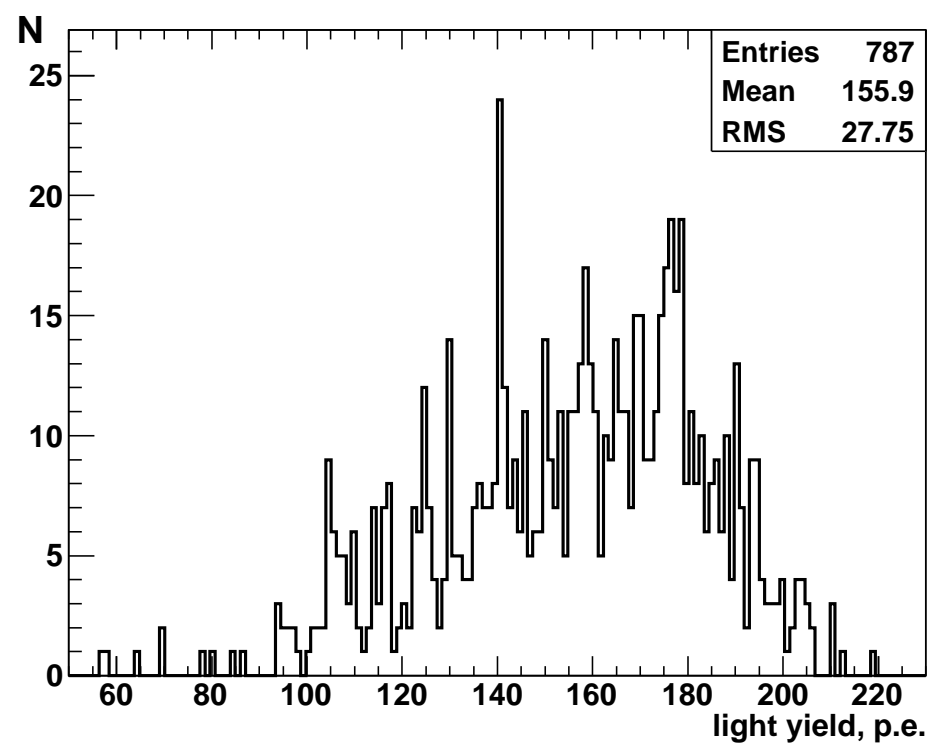

Figure 6: The light yield distribution of manufactured EMMA counters.

ufactured counters have the light yield of more than 100 p.e. and satisfy the requirements of the experiment.

\section{Conclusion}

The MRS APD's manufactured for the EMMA experiment have high PDE values and show a low temperature dependence of the main parameters. The scintillator counters with WLS fiber 
readout and MRS APD's provide a high light yield and a good time resolution. The production of all counters will be finished in December 2009. Installation of all modules and commissioning of the detector is expected in the first half of 2010.

It is a pleasure for authors to thank the INR technicians for their excellent work on the preparation and assembly of the detectors. The work of the authors from the University of Oulu is funded by the European Union Regional Development Fund and is also supported by the Academy of Finland (Project 108991).

\section{References}

[1] T. Enqvist et.al., Nucl. Phys. B (Proc. Suppl.) 165 (2007) 349; T. Enqvist et.al., Nucl. Phys. B (Proc. Suppl.) 175-176 (2008) 307.

[2] V. Golovin et.al., Patent for invention in Russia, No. 1644708, 1989; A.G. Gasanov et al., Lett. J. Techn. Phys. 16 (1990) 14 (in Russian).

[3] G. Bondarenko et al., Nucl. Instr. Meth., A442 (2000) 187.

[4] E.V. Akhrameev et al., arXiv:0901.4675 [physics.ins-det].

[5] Yu.V. Musienko et al., Instrum. Exp. Tech. 51 (2008) 101.

[6] M. Yokoyama et al., arXiV:0807.3145 [physics.ins-det].

[7] F. Retiere, Using MPPC's for T2K near detector, talk at this Workshop.

[8] Yu.Kudenko et al., PoS PD07:016, 2007.

[9] V.I. Volchenko et al., arXiV:0810.2414 [physics.ins-det].

[10] Y. Du and F. Retiere, Nucl. Instr. Meth. A596 (2008) 396.

[11] O. Mineev et al., Nucl. Instr. Meth. A577 (2007) 540; A.Izmaylov et al., arXiv:0904.4545.

[12] "T2K ND280 Conceptual Design Report", T2K Internal Document; D. Karlen, Nucl. Phys. B (Proc. Suppl.) 159 (2006) 91; Yu.G. Kudenko, Nucl. Instrum. Meth. A598 (2009) 289.

[13] Yu.G. Kudenko et al., Nucl. Instr. Meth. A469 (2001) 340; O. Mineev et al., Nucl. Instr. Meth. A494 (2002) 362; N. Yershov et al., Nucl. Instr. Meth. A543 (2005) 454. 